International Journal of Electrical Engineering and Technology (IJEET)

Volume 11, Issue 4, June 2020, pp. 30-44, Article ID: IJEET_11_04_004

Available online at https://iaeme.com/Home/issue/IJEET? Volume $=11 \&$ Issue $=4$

ISSN Print: 0976-6545 and ISSN Online: 0976-6553

DOI: https://doi.org/10.34218/IJEET.11.4.2020.004

(C) IAEME Publication

Scopus Indexed

\title{
IMPLEMENTATION OF ANFIS ALGORITHM FOR EFFICIENT SPECTRUM HANDS-OFFS IN COGNITIVE RADIO NETWORKS
}

\author{
Meghana S \\ Department of Telecommunication, \\ RV College of Engineering, Bangalore, India \\ Dr. B. Roja Reddy \\ Associate Professor, Department of Telecommunication, \\ RV College of Engineering, Bangalore, India
}

\begin{abstract}
The advancements in wireless communication in the recent times have raised the demand for wireless radio spectrum. Thus, Cognitive Radio Network (CRN) is introduced as it has the capability to sense the available channels and radio-frequency $(R F)$ spectrum in order to minimize the interference to primary users. Basically, Cognitive Radio $(C R)$ is the network that alters the parameters of transmission and enables more users to communicate concurrently. However, unnecessary handovers in the wireless network lead to the rise of ping pong effect. This effect reduces the efficiency of the network in terms of providing continuous call to the users. The conventional fuzzy model used two fuzzy controllers to implement the entire process. Although, it is efficient but this model is very complex as Fuzzy rules are generated manually. Thus, a novel approach is presented in this dissertation in which amalgamation of fuzzy inference system and neural network is carried out. The main purpose of designing this approach is to reduce the probability of Spectrum Hands-off (HO) which in turn will reduce the ping pong effect. The proposed approach includes two ANFIS controllers and the implementation is performed in terms of different parameters such as Signal Strength from primary to secondary user (SSps), Signal to noise ratio (SNRpu), power of secondary user (Psu), Channel hold time (HT), Velocity of secondary user (Vsu). Simulation is carried out in MATLAB and the comparative analysis is performed which ensured the effectiveness of the proposed ANFIS model. It is observed that the outcome of proposed model surpassed the efficiency of conventional fuzzy logic controller.
\end{abstract}

Key words: Cognitive Radio, Ping Pong, Fuzzy, ANFIS, Spectrum Handoff. 
Cite this Article: Meghana S and Dr. B. Roja Reddy, Implementation of ANFIS Algorithm for Efficient Spectrum Hands-offs in Cognitive Radio Networks, International Journal of Electrical Engineering and Technology, 11(4), 2020, pp. 30-44.

https://iaeme.com/Home/issue/IJEET?Volume $=11 \&$ Issue $=4$

\section{INTRODUCTION}

The development in wireless correspondence and systems administration has expanded the interest for the accessibility of additional spectrum bands. Research in the domain has envisaged that the issue of spectrum scarcity can be addressed by Cognitive Radio (CR). Cognitive Radio Networks (CRNs) are comprised of multiple technologies and networks which permits the dynamic use of spectrum. Spectrum Handoff (HO), is thus a major necessity of Cognitive Radio Network and if not properly executed, might cause ping-pong effect [1]. When mobile users move between the boundaries of adjacent cells, it leads to needless Spectrum HOs that leads to rise of Ping pong effect. Fuzzy Logic based framework is recommended that is fit for settling on proficient choices about executing Spectrum HOs along these lines, and diminishes the odds of the ping-pong impact. It is commonly believed that there is a spectrum scarcity at frequencies that can be economically used for wireless communications. The strong competition for spectra use at frequencies less than $3 \mathrm{GHz}$ has given rise the concern for seamless HO. Licensed user or Primary user (PU), is a licensed user who has the priority to use the spectrum, Cognitive user or Secondary user (SU), accesses the network opportunistically in the absence of licensed user, it is thus important for the SU to be intelligent enough to sense the availability of spectrum bands. Spectrum sensing involves sensing the surrounding RF frequencies which are not currently in use of PU [2]. The spectrum handoffs, is the process where a SU switches its operating frequency because of the many reasons including channel occupation by the PU, sudden arrival of PU, quality of service degradation, interference to the transmission of PU etc. Cognitive Radio (CR) is a flexible, smart radio and network technology that senses available channels dynamically within a wireless spectrum and adjusts transmission parameters to make it possible for more communication to function simultaneously, as well as enhanced radio operating behaviour. There are no active primary users for these elements, called white space as shown in figure 1, within these parts of the spectrum, secondary users can freely detect and communicate and the method is referred to as an overlay approach which is taken into account in many radio systems [3]. The power level is also very small in the other areas of the continuum. By adding cognitive radio capabilities, spectrum can be used as a basis, provided it is regulated under a predefined threshold level of the transmitting power of the secondary users has no effect on Primary users. To solve non regular distribution of spectrum hands off back channel selection method has been suggested basically spectrum hands off occur when the PUs arrive in the channel, therefore the cognitive user has the leave the channel, the transmission will be interrupted hence the backup channel approach is suggested, it will give the another free channel to resume the cognitive user transmission [4]. Proactive spectrum hands off is mainly used to avoid the interference between the PUs and SUs by detecting the mobility of the PU and initiating the spectrum hands off before the Pus reappear hence it helps achieving the personal gain [5]. For increasing the efficiency of the network probability-based spectrum hands off with centralized method are used, it provides the accuracy for sensing the right channel for hands off also reduces the energy required, hands off and speed up the transmission of the data [6]. Spectrum management is the important task of the CRN, it helps the SUs to get benefit from the spectrum without interference with the Pus by using the pure reactive hands-off method [7]. 


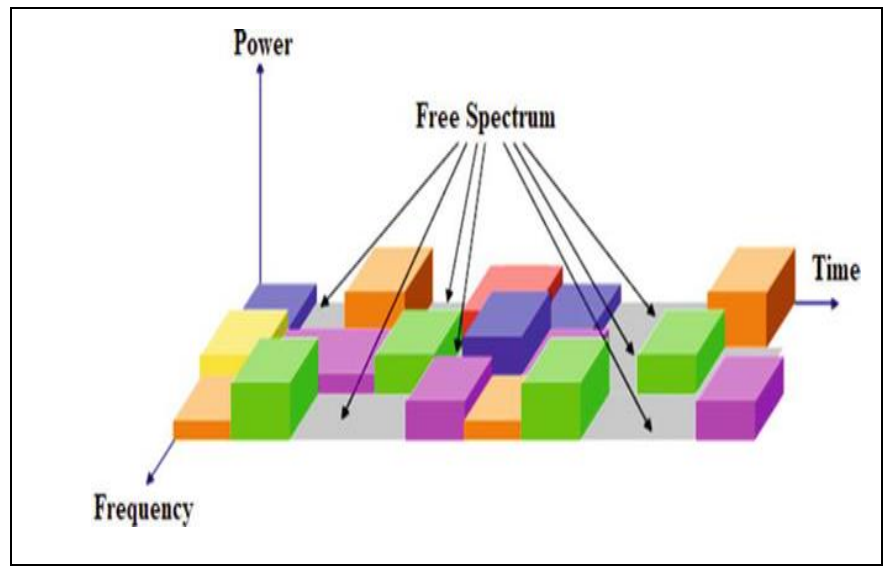

Figure 1 The free and occupied spectrums [1]

When Spectrum Allocation is performed, most of the time $90 \%$ of the Spectrum Remains unused hence in the CRN, Licensed user who has the priority on the spectrum band, in the absence of the licensed user, unlicensed user can sense the availability of the spectrum band for transmission [8]. The sudden arrival of the primary user (licensed) disrupts the Secondary user (unlicensed) transmission, it will cause the degradation pf the quality of the service (QOs), hence in order to avoid this problem, spectrum handoff's (SH) are required. The four main functions of the SH are Spectrum Sensing, Channel Selection, Spectrum Sharing and Spectrum Mobility.

\section{SYSTEM MODEL}

This section discusses about the ping pong effect and two algorithms to overcome this effect in cognitive radio. The algorithms are conventional fuzzy model used two fuzzy controllers to implement the entire process and a novel approach which uses amalgamation of fuzzy inference network and neural network.

\subsection{Ping Pong Effect in Cognitive Radio}

The Ping-pong effect happens when a mobile station (MS) performs HOs frequently and rapidly between base stations (BS) when the signal strength from the previous BS is sufficient. For the purpose of resource management and due to unpredictable nature of wireless communication, some intelligent technique is required that can facilitate in deciding about carrying out of Spectrum HOs. One such technique is the Fuzzy logic and ANFIS Algorithm. Fuzzy logic helps in modelling problems where traditional mathematical methods cannot be applied effectively or where the information about the problem is not complete. From the figure 2, CR Network is considered which is assumed to be composed of two networks, i.e. UMTS and WLAN, furthermore, SUs is assumed to have the ability to sense the presence of PUs nearby. The system consists of two sets of Fuzzy inference Logic Controllers such as FLC-1 and FLC-2 respectively. The first system i.e. FLC-1 is designed to control the power of SU, so that interference to PU can be controlled and kept to its minimum. The second system i.e. FLC-2 is designed to take decision in order to execute Spectrum HOs. Inputs to FLC-2 are Channel Hold-time (HT), Velocity of SU ( $\left.\mathrm{V}_{\mathrm{SU}}\right)$ and power of SU (PSU) [9]. The occurrence of Spectrum HOs in an environment. Where conditions and situations of the network are ambiguous and unknown, the range of transmitting power of SU is kept between that of WLAN and UMTS i.e. $30 \mathrm{dBm}$ and $43 \mathrm{dBm}$ respectively [10]. The method of Defuzzification used is centroid. 


\subsection{Fuzzy Inference Algorithm}

Fuzzy Inference algorithm is developed by using fuzzy if-then rule, and these fuzzy if-then rule are also called the linguistic variables using if $\mathrm{X}$ and Then $\mathrm{Y}$, where $\mathrm{X}$ and $\mathrm{Y}$ represents the Fuzz sets characterized by the Membership variables, mainly these rules are used to identify the non-accurate and unknown variations that exists when the decision has be taken at the output and its flowchart as shown in the figure 3.

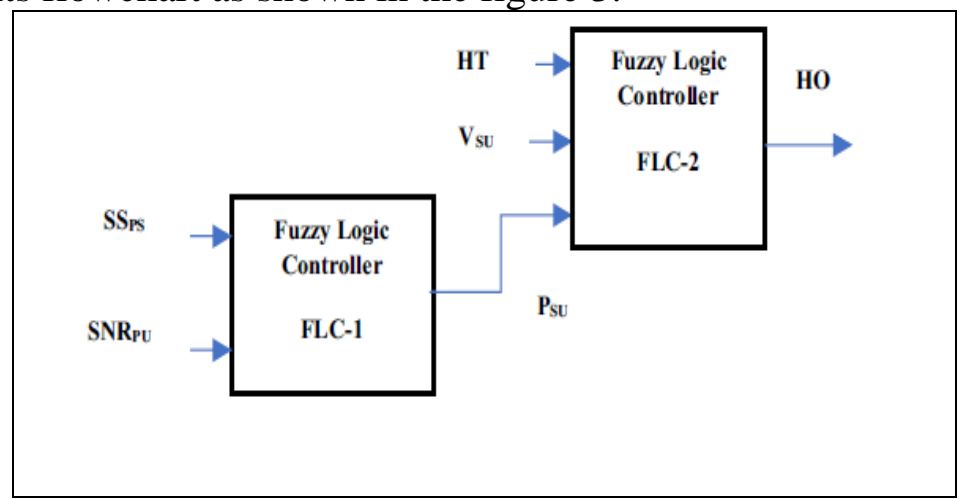

Figure 2 Fuzzy Logic Controller [3]

\subsection{ANFIS Algorithm}

Table 1 Rule base for FLC

\begin{tabular}{|c|c|c|c|c|}
\hline \multicolumn{4}{|c|}{ Inputs } & \multirow{2}{*}{$\begin{array}{l}\text { Output } \\
\text { HO }\end{array}$} \\
\hline Rules & HT & $\mathbf{P}_{\mathrm{SF}}$ & $\mathbf{V}_{\mathbf{S F}}$ & \\
\hline 1 & Low & Low & Low & Medium \\
\hline 2 & Low & Low & Medium & High \\
\hline 3 & Low & Low & High & Very High \\
\hline 4 & Low & Medium & Low & Medium \\
\hline 5 & Low & Medium & Medium & High \\
\hline 6 & Low & Medium & High & High \\
\hline 7 & Low & High & Low & High \\
\hline 8 & Low & High & Medium & High \\
\hline 9 & Low & High & High & Very High \\
\hline 10 & Medium & Low & Low & \\
\hline 11 & Medium & Low & Medium & Medium \\
\hline 12 & Medium & Low & High & High \\
\hline 13 & Medium & Medium & Low & Low \\
\hline 14 & Medium & Medium & Medium & Low \\
\hline 15 & Medium & Medium & High & High \\
\hline 16 & Medium & High & Low & Very Low \\
\hline 17 & Medium & High & Medium & Low \\
\hline 18 & Medium & High & High & dium \\
\hline 19 & High & Low & Low & Medium \\
\hline 20 & High & Low & Medium & High \\
\hline 21 & High & Low & High & Very High \\
\hline 22 & High & Medium & Low & Very Low \\
\hline 23 & High & Medium & Medium & Low \\
\hline 24 & High & Medium & High & Medium \\
\hline 25 & High & High & Low & Very Low \\
\hline 26 & High & High & Medium & Low \\
\hline 27 & H11 & High & High & Medium \\
\hline
\end{tabular}


ANFIS algorithm is based on FIS and it is additionally known as Adaptive Neuro-Fuzzy Inference System and it could be a Hybrid system that can merge the Artificial Neural Network \& Conventional Fuzzy logic system by upsurging the performance of each ways of AI then reduces the drawback of technique to get an adaptive System that gives the higher result and intelligence compared with both of the Method one by one. Due to Incalculability of the handshaking procedure in the mobile network and improper nature of those factors, ANFIS methodology being employed to check the changes with those paraments over a amount of time then train the network to form the hands-off probability decision based on the changes Identified while training the data [13]. ANFIS is associate adaptive network of ANN and FIS integrating the power of neural network to supervise learning technique and it works on live input and output knowledge to get fuzzy membership rules.

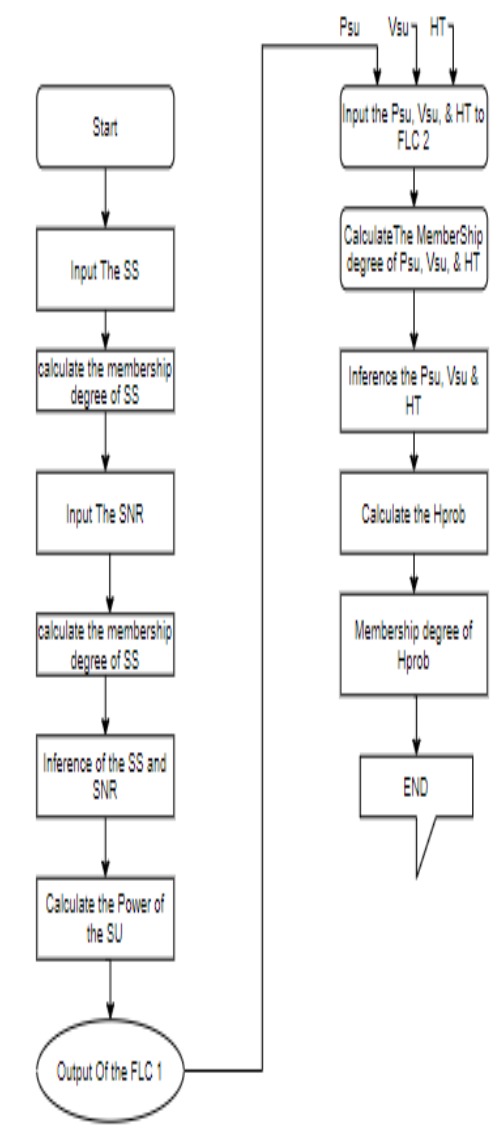

Figure 3 Flowchart of the Fuzzy inference network

From the table 1, the rule base for Fuzzy logic controller, the probability of Spectrum HOs with respect to $P_{S U}$ and $V_{S U}$. Figure shows that probability of Spectrum HOs remains very low even for high values of $\mathrm{P}_{\mathrm{SU}}$. When the value of $\mathrm{V}_{\mathrm{SU}}$ is maximum, means that probability of Spectrum HOs is high only when the value of PSU is low and that of $V_{\text {SU }}$ is high [14]. probability of Spectrum HOs decreases and velocity of SU ( $\left.\mathrm{V}_{\mathrm{SU}}\right)$ increases, the probability of Spectrum HOs also increases but that increase in $\mathrm{HO}_{\text {prob }}$ depends on the status of Channel hold-time thus, $\mathrm{HO}_{\text {prob }}$ is controlled by $\mathrm{HT}$ therefore, chances of ping-pong effect at high velocity becomes less.

Hence the conventional Fuzzy logic controller and ANFIS algorithm are used for efficient execution of spectrum handoff in the CRNs, thereby reduces the ping pong effec 


\section{IMPLEMENTATION AND MECHANISM}

The novel technique is designed to compute the handover probability in order that ping pong effect can be reduced. In the previous work, two fuzzy logic controllers are used to determine the HO probability. And the performance was analysed using different parameters for each case. In the proposed work, fuzzy systems are replaced by ANFIS model due to its following advantages:

- ANFIS is the amalgamation of neural network and fuzzy inference system which makes it an intelligent model. The features of neural network enable this model to analyze the data deeply and precisely.

- It eliminates the rule generation for different situation as ANFIS offers the feature of self-optimization.

- It performs operation better than that of fuzzy logic

Moving toward its implementation, the entire process was performed in two phases. Two ANFIS controllers are designed. These phases are delineated below for both previously designed fuzzy logic systems and Proposed ANFIS model.

\subsection{Implementation of First Controller}

In the first phase, two inputs -SSps and SNRpu are given to the fuzzy system to compute Psu. These parameters are as explained as follows, SSps is the signal strength of primary user (PU) as measured by secondary user (SU). SNRpu is the signal to noise ratio of primary user. This controller is designed to achieve the output Psu. The output is attained in term of power of the secondary user. The GUI designed for phase 1 has two options to compute the output i.e. power of SU. The output is determined for both existing model (Fuzzy) and proposed model (Neuro Fuzzy). Different values are taken into consideration to determine the power of secondary user. Strength from secondary to primary user varies from -92 to 0 and for signal to noise ratio of primary user it has the range from 0 to 28 . These values are kept same for both existing and proposed model in order that the performance of the systems will be determined on this basis. The design of ANFIS model is represented in figure 5. Sugeno type fuzzy is used for the ANFIS model. The inputs SSps and SNRpu are given to the ANFIS where 9 rules are generated. These rules are generated by ANFIS according to the different data retrieved by it. Eventually, the output is received in terms of power of secondary user Psu.

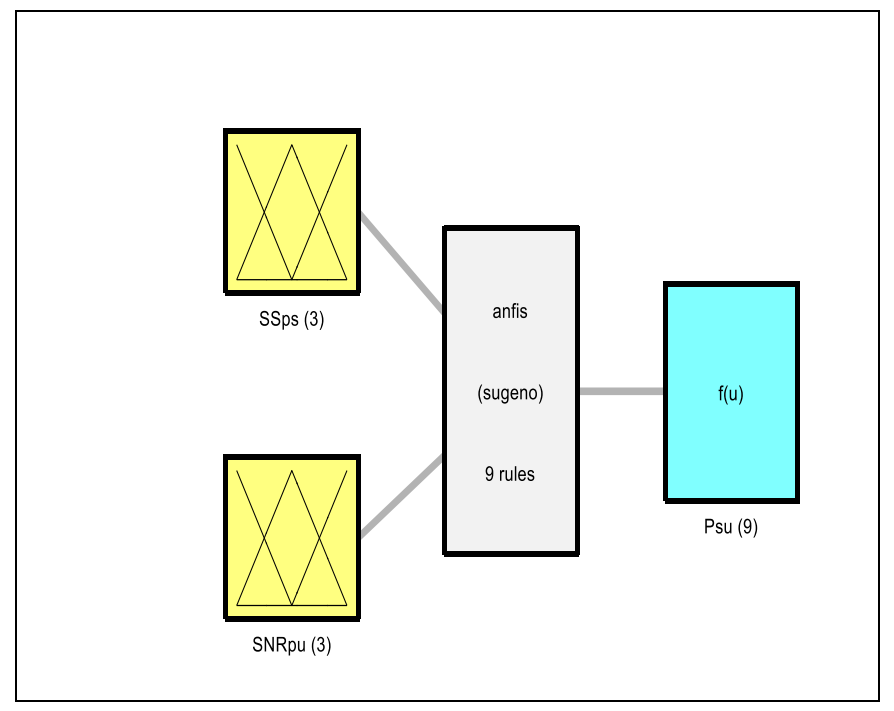

Figure 4 Proposed ANFIS model for first phase 


\subsection{Implementation of Second Phase}

The out obtained by the first ANFIS model is taken as the input for second phase. It is further used to compute the handover probability of the Cognitive Radio network. In order to do so, two more parameters are taken along with Psu. These are as follows:

HT is the channel hold time. It is referred to the time when the channel is not being utilized by the primary user (PU).

Vsu Is the velocity of the secondary user and Psu is the output of first controller.

These three parameters act as the input to the second controller.

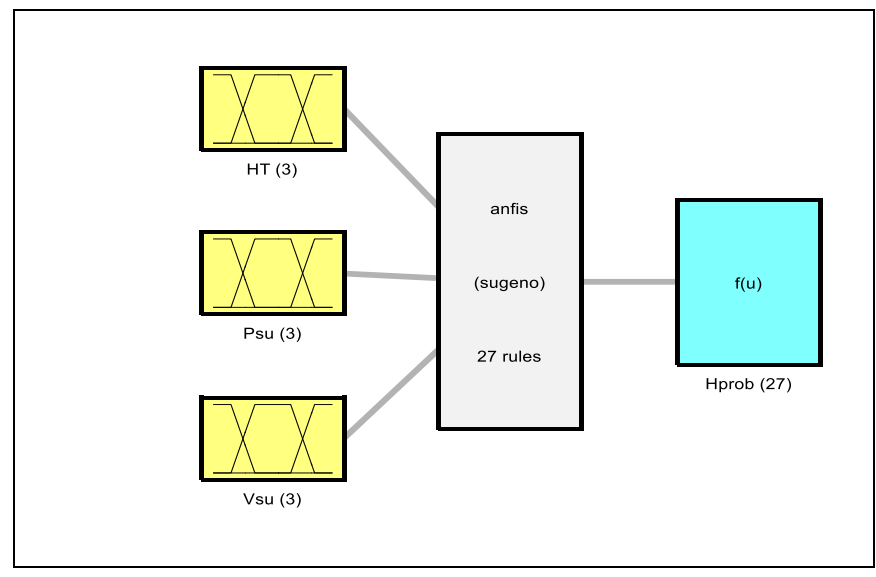

Figure 5 Proposed model for ANFIS used for second controller

The architectural design for proposed ANFIS controller is presented in figure 5. It can be seen that three inputs are given for the Sugeno Fuzzy type-based controller. Neural network enabled the model to generate 27 rules and eventually Hprob is obtained as the final output. The above diagram explains the flow of data in the ANFIS controller. In order to calculate the HO probability for proposed model, the GUI is used where the output is computed just by clicking the button. Figure 6 displayed the snapshot while computing probability of HO. Different iterations are performed to obtain the values in different scenarios. The GUI to compute the HO probability (HOprob) has the values of the input can be attuned according to the requirement, however, to compute the values for existing system and proposed system, two buttons are given. Firstly, the membership functions are generated to use than as the input to fuzzy based controller. The degree of membership is defined in low, medium and high level. The range of each parameter is recorded in the following table

Table 2 Range of parameters

\begin{tabular}{|c|c|c|}
\hline Sl. No & Parameter & Range \\
\hline 1 & HT & $0-1$ \\
\hline 2 & Psu & $0-45$ \\
\hline 3 & Vsu & $0-100$ \\
& & $\mathrm{Km} / \mathrm{h}$ \\
\hline
\end{tabular}




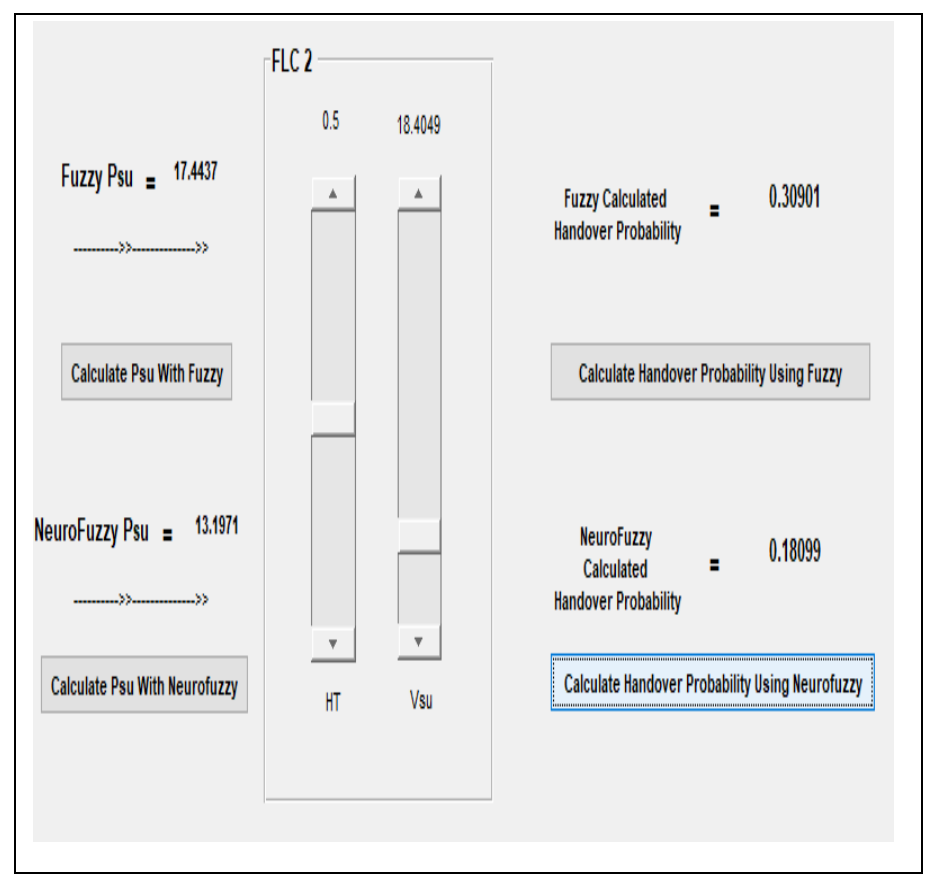

Figure 6 GUI to compute output for proposed model

\section{RESULTS}

The simulated results are discussed for the conventional fuzzy model and an algorithm which uses amalgamation of fuzzy inference system and neural network.

\subsection{Results of Conventional Fuzzy Logic Controller}

The results obtained by the designed model are explained in detail and comparative analysis is performed in order to validate the efficacious performance of the proposed ANFIS controller to reduce unnecessary HOs and rising of the ping pong effect. The results are presented in the 3D graphical view for easy demonstration.

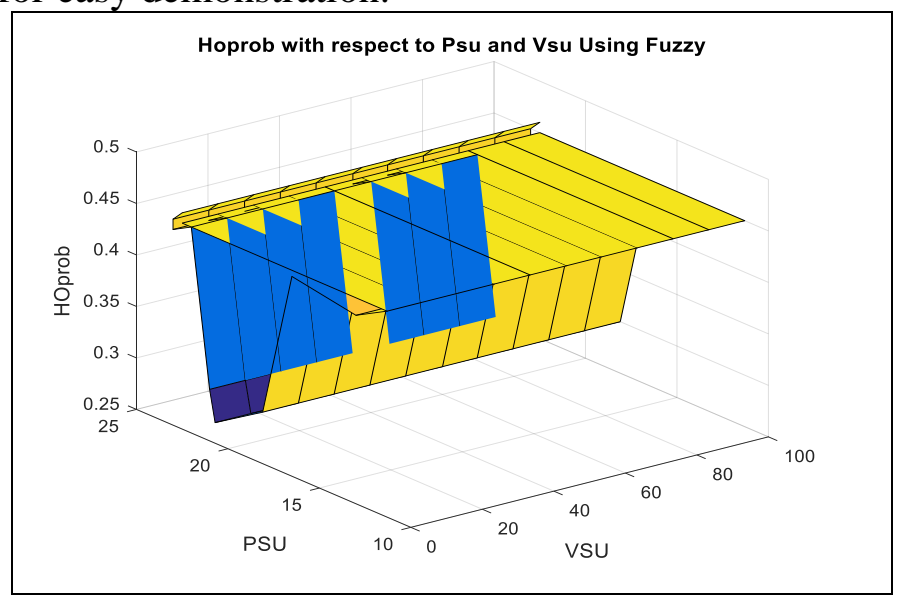

Figure $7 \mathrm{HO}$ probability in terms of Psu and Vsu using fuzzy model

Figure 7 presents the 3D graph for HO probability in terms of Psu and Vsu. The graph reveals that $\mathrm{HO}$ probability is obtained for 11 iterations i.e. 11 different values of Psu and Vsu. The maximum HO probability constituted to 0.46 at $100 \mathrm{~km} / \mathrm{h}$ and minimum $\mathrm{HO}$ 
probability accounted to 0.27 at $50.3 \mathrm{~km} / \mathrm{h}$ velocity of secondary user. The corresponding values of the parameters are recorded in table 3 . The value of HO probability for conventional work varies from 0.27 to 0.46 . It is observed as for most of the cases, the probability remains between 0.43 to 0.46 .

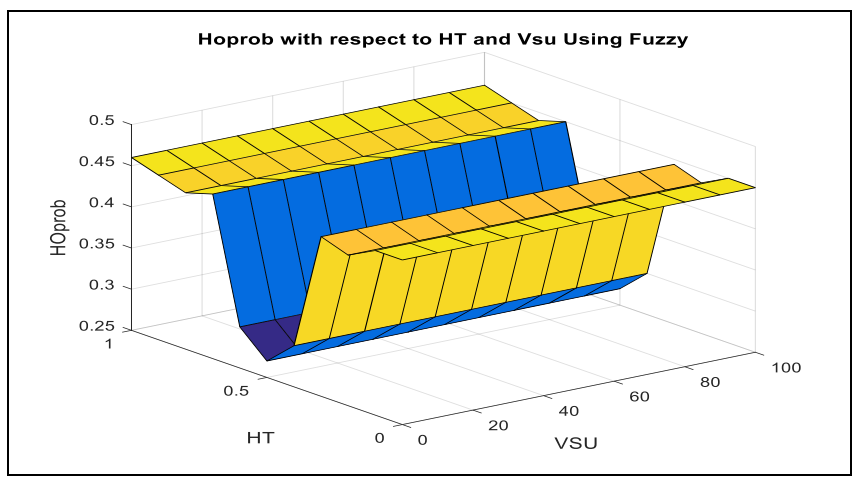

Figure $8 \mathrm{HO}$ probability in terms of $\mathrm{HT}$ and Vsu using fuzzy model

Figure 8 demonstrates the graph showing the HO probability in terms of hold time and velocity of secondary users. The graph shows the probability of HO is high in the beginning and it reduced till $6^{\text {th }}$ iteration. After this, the probability experiences a gradual increase it its value. The retrieved values of Ho probability are presented in the table 3 . The HO probability for the conventional fuzzy controller is observed to better. Further, in the subsequent section, the likelihood of HO for proposed system is explained.

\subsection{Results of the ANFIS Controller}

The proposed ANFIS controller reduced the complexity of the computing the HO probability in terms of different factors. The analysis of output is presented with respect to the (Psu, Vsu) and (HT, Vsu). In figure 9, the representation of $3 \mathrm{~d}$ graph is displayed for power and velocity of the secondary user. The obtained probability suggests that when power and velocity is 0 then the likelihood of the handover occur accounted to 0.4. This number is the maximum probability of $\mathrm{HO}$ and the minimum $\mathrm{HO}$ probability constituted to 0.17 . The output is taken for 11 iterations in which the power and the velocity is always different. The obtained data is recorded in a tabular form shown in table 4.

Table 3 HO probability for conventional fuzzy system in terms of Psu and Vsu, HT and Vsu

\begin{tabular}{|c|c|c|c|}
\hline $\begin{array}{c}\text { SI. } \\
\text { no. }\end{array}$ & $\begin{array}{c}\text { PSU } \\
\text { fuzzy }\end{array}$ & VSU & Fuzzy ho \\
\hline 1 & 22.5 & 0 & 0.45 \\
\hline 2 & 11.3 & 10 & 0.45 \\
\hline 3 & 13 & 20 & 0.433 \\
\hline 4 & 16.5 & 30 & 0.444 \\
\hline 5 & 18.1 & 40 & 0.3 \\
\hline 6 & 20.7 & 50.3 & 0.27 \\
\hline 7 & 21 & 60 & 0.3 \\
\hline 8 & 22 & 70 & 0.45 \\
\hline 9 & 23 & 80 & 0.44 \\
\hline 10 & 23 & 90 & 0.45 \\
11 & 22.5 & 100 & 0.46 \\
\hline
\end{tabular}

\begin{tabular}{|c|c|c|c|}
\hline $\begin{array}{c}\text { SI. } \\
\text { no. }\end{array}$ & HT & VSU & $\begin{array}{c}\text { Fuzzy HO } \\
\text { Probability }\end{array}$ \\
\hline 1 & 0 & 0 & 0.45 \\
\hline 2 & 0.1 & 10 & 0.45 \\
\hline 3 & 0.2 & 20 & 0.433 \\
\hline 4 & 0.3 & 30 & 0.444 \\
\hline 5 & 0.4 & 40 & 0.3 \\
\hline 6 & 0.5 & 50.3 & 0.27 \\
\hline 7 & 0.6 & 60 & 0.3 \\
\hline 8 & 0.7 & 70 & 0.45 \\
\hline 9 & 0.8 & 80 & 0.44 \\
\hline 10 & 0.9 & 90 & 0.45 \\
\hline 11 & 1 & 100 & 0.46 \\
\hline
\end{tabular}


Implementation of ANFIS Algorithm for Efficient Spectrum Hands-offs in Cognitive Radio Networks

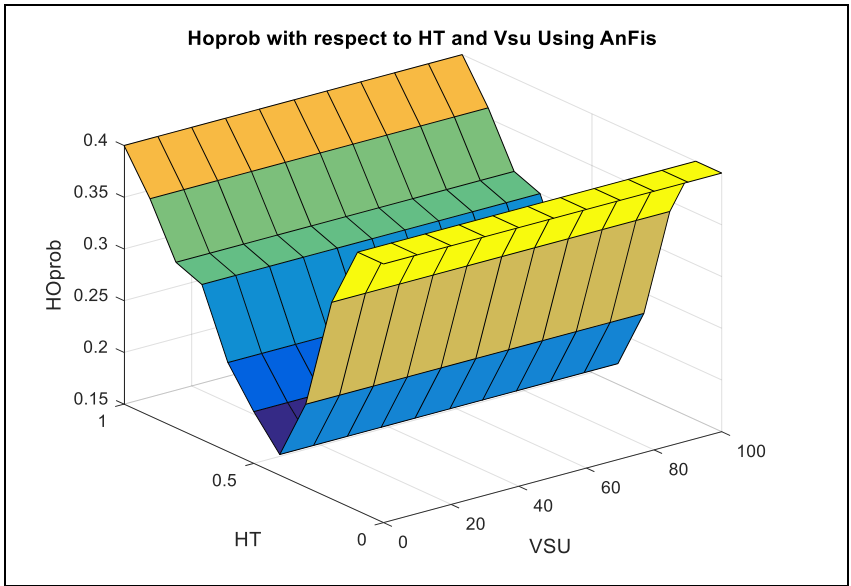

Figure $9 \mathrm{HO}$ probability in terms of Psu and Vsu using ANFIS model

Table 4 HO probability for proposed ANFIS system in terms of Psu and Vsu, HT and Vsu

\begin{tabular}{|c|c|c|c|}
\hline $\begin{array}{c}\text { Sl. } \\
\text { no. }\end{array}$ & HT & VSU & $\begin{array}{c}\text { ANFIS } \\
\text { HO }\end{array}$ \\
\hline 1 & 0 & 0 & 0.4 \\
\hline 2 & 0.1 & 10 & 0.4 \\
\hline 3 & 0.2 & 20 & 0.34 \\
\hline 4 & 0.3 & 30 & 0.23 \\
\hline 5 & 0.4 & 40 & 0.17 \\
\hline 6 & 0.5 & 50.3 & 0.2 \\
\hline 7 & 0.6 & 60 & 0.236 \\
\hline 8 & 0.7 & 70 & 0.3 \\
\hline 9 & 0.8 & 80 & 0.31 \\
\hline 10 & 0.9 & 90 & 0.36 \\
\hline 11 & 1 & 100 & 0.4 \\
\hline
\end{tabular}

\begin{tabular}{|c|c|c|c|}
\hline $\begin{array}{c}\text { SI. } \\
\text { no. }\end{array}$ & $\begin{array}{c}\text { PSU } \\
\text { Anfis }\end{array}$ & VSU & $\begin{array}{c}\text { ANFIS } \\
\text { HO }\end{array}$ \\
\hline 1 & 0 & 0 & 0.4 \\
\hline 2 & 3.3 & 10 & 0.4 \\
\hline 3 & 5.7 & 20 & 0.34 \\
\hline 4 & 11.54 & 30 & 0.23 \\
\hline 5 & 14.7 & 40 & 0.17 \\
\hline 6 & 22 & 50.3 & 0.2 \\
\hline 7 & 24 & 60 & 0.236 \\
\hline 8 & 29 & 70 & 0.3 \\
\hline 9 & 35 & 80 & 0.31 \\
\hline 10 & 41 & 90 & 0.36 \\
\hline 11 & 45 & 100 & 0.4 \\
\hline
\end{tabular}

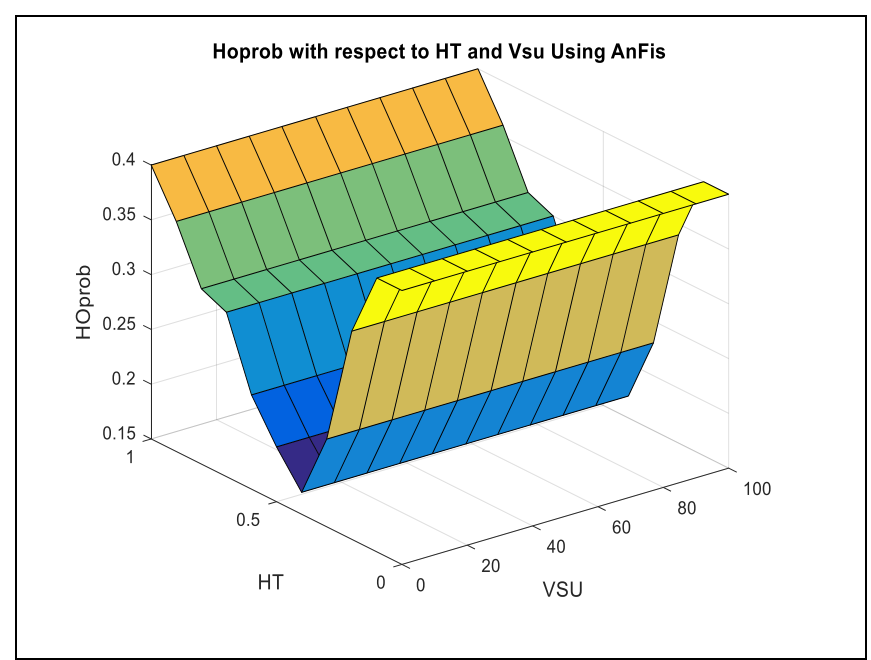

Figure $10 \mathrm{HO}$ probability in terms of HT and Vsu using ANFIS model 
Similarly, for HT and Vsu, the HO probability is demonstrated in the above graph (figure 10). The values of $\mathrm{HO}$ are shown in table 4 . The results reveal that the Ho probability experience a downward parabola shape i.e. high in the beginning that gradually decreased when coming to the iteration in the middle and further, increase is experienced till last iteration.

\section{COMPARATIVE ANALYSIS OF PROPOSED AND CONVENTIONAL TECHNIQUES}

This section presents the comparative analysis of handover probability with respect to velocity (Vsu), power (Psu) of secondary user and channel hold time (HT). For different values of velocity, the graph is plotted for $\mathrm{HO}$ probability that is delineated in figure 11 . The graph presents the velocity on $\mathrm{x}$ axis ranging from $0-100 \mathrm{~km} / \mathrm{h}$ and probability on $\mathrm{Y}$ axis. The outcome of proposed ANFIS model is observed to be less as compared to that of the conventional method. When velocity is $100 \mathrm{~km} / \mathrm{h}$ the likelihood of HO for proposed work is 0.4 whereas for conventional technique it accounted to 0.46 which is higher.

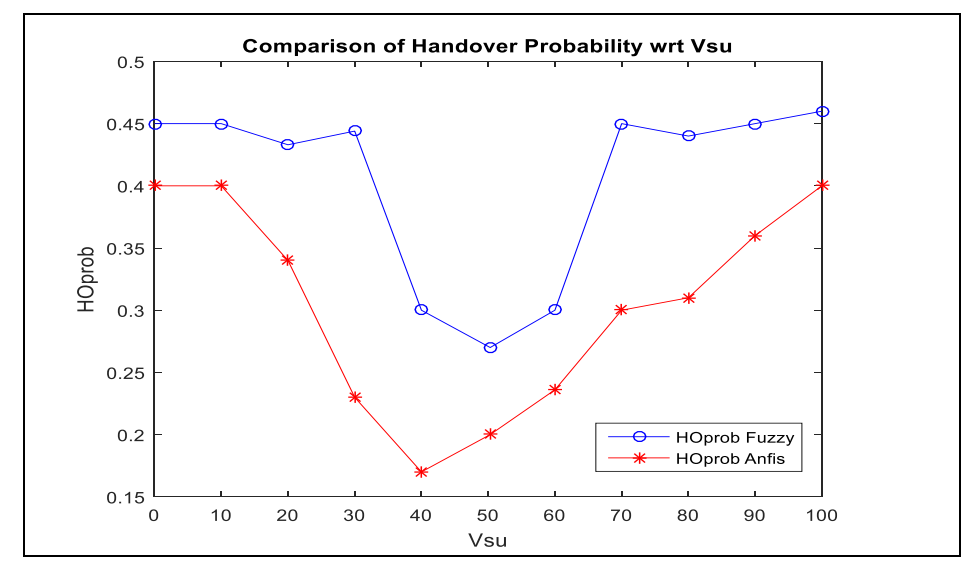

Figure 11 Comparative analysis of $\mathrm{HO}$ probability in terms of Vsu

The more the probability the more will be ping pong effects. A great difference can be seen in the probability for proposed and existing system which ensured the effectiveness of NFIS model. The values are recorded in the following table (Table 5).

Table 5 Comparison of proposed and existing system HO probability wrt Vsu

\begin{tabular}{|c|c|c|}
\hline VSU & Fuzzy HO & ANFIS HO \\
\hline 0 & 0.45 & 0.4 \\
\hline 10 & 0.45 & 0.4 \\
\hline 20 & 0.433 & 0.34 \\
\hline 30 & 0.444 & 0.23 \\
\hline 40 & 0.3 & 0.17 \\
\hline 50.3 & 0.27 & 0.2 \\
\hline 60 & 0.3 & 0.236 \\
\hline 70 & 0.45 & 0.3 \\
\hline 80 & 0.44 & 0.31 \\
\hline 90 & 0.45 & 0.36 \\
\hline 100 & 0.46 & 0.4 \\
\hline
\end{tabular}




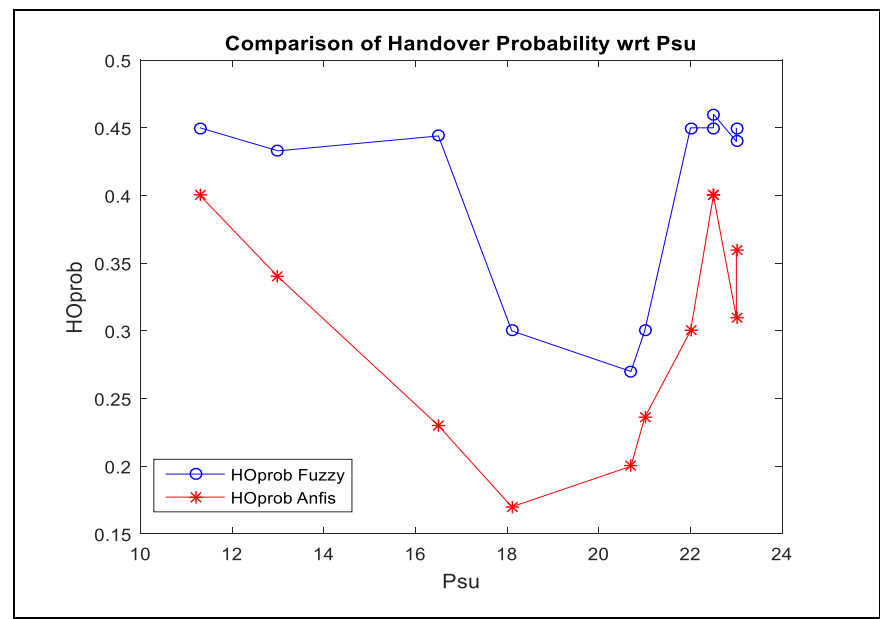

Figure 12 Comparative analysis of Ho probability in terms of Psu

Table 6 Comparison of proposed and existing system HO probability wrt Psu

\begin{tabular}{|c|c|c|c|}
\hline $\begin{array}{c}\text { PSU } \\
\text { fuzzy }\end{array}$ & $\begin{array}{c}\text { PSU } \\
\text { Anfis }\end{array}$ & $\begin{array}{c}\text { Fuzzy } \\
\text { HO }\end{array}$ & $\begin{array}{c}\text { ANFIS } \\
\text { HO }\end{array}$ \\
\hline 22.5 & 0 & 0.45 & 0.4 \\
\hline 11.3 & 3.3 & 0.45 & 0.4 \\
\hline 13 & 5.7 & 0.433 & 0.34 \\
\hline 16.5 & 11.54 & 0.444 & 0.23 \\
\hline 18.1 & 14.7 & 0.3 & 0.17 \\
\hline 20.7 & 22 & 0.27 & 0.2 \\
\hline 21 & 24 & 0.3 & 0.236 \\
\hline 22 & 29 & 0.45 & 0.3 \\
\hline 23 & 35 & 0.44 & 0.31 \\
\hline 23 & 41 & 0.45 & 0.36 \\
\hline 22.5 & 45 & 0.46 & 0.4 \\
\hline
\end{tabular}

Similarly, the graph is obtained for power of secondary user and it is shown in figure 12 . The values achieved for his parameter is different for proposed and convention technique. The graph shows the Psu on $\mathrm{x}$ axis that has a range from 0 to 24 . However, Psu is achieved by giving same values of SSps and SNRpu to ANFIS and fuzzy controller. In the graph, he differences between the outputs can be seen. The highest probability of $\mathrm{HO}$ is 0.46 for conventional, however it is 0.4 for proposed technique. Low HO probability makes it more effective than that of previously existing technique.

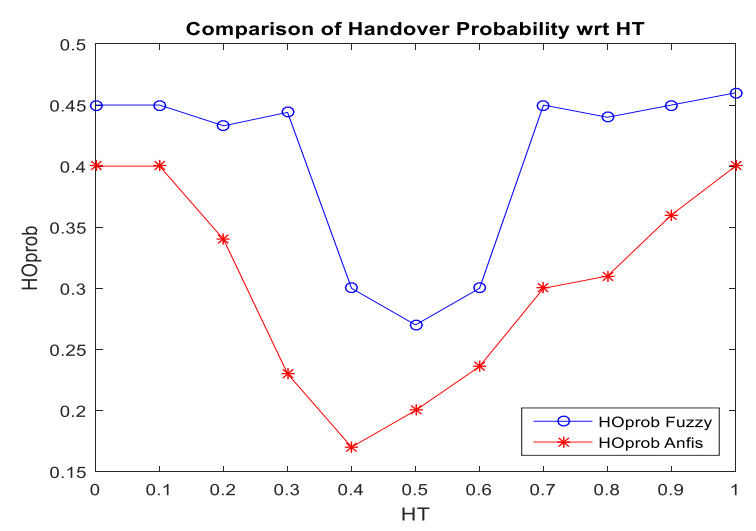

Figure 13 Comparative analysis of HO probability in terms of HT 
Further, the last comparison is performed between conventional and proposed technique with respect to the channel hold time. HT is presented on $\mathrm{x}$-axis and has range from 0 1 second and it is shown in figure 13 . The HO probability doesn't exceed 0.5 in both the cases. The minimum likelihood of HO accounted to 0.17 for proposed ANFIS model where for fuzzy controller it constituted to 0.27 . For every iteration of HT. The probability for the proposed work is less than that of conventional technique. From this comparison, it is observed that the proposed technique is capable of reducing the HO probability in cognitive radio network. The remaining values got hold time are shown in table 7.

Table 7 Comparison of proposed and existing system HO probability wrt Vsu

\begin{tabular}{|c|c|c|}
\hline HT & Fuzzy HO & ANFIS HO \\
\hline 0 & 0.45 & 0.4 \\
\hline 0.1 & 0.45 & 0.4 \\
\hline 0.2 & 0.433 & 0.34 \\
\hline 0.3 & 0.444 & 0.23 \\
\hline 0.4 & 0.3 & 0.17 \\
\hline 0.5 & 0.27 & 0.2 \\
\hline 0.6 & 0.3 & 0.236 \\
\hline 0.7 & 0.45 & 0.3 \\
\hline 0.8 & 0.44 & 0.31 \\
\hline 0.9 & 0.45 & 0.36 \\
\hline 1 & 0.46 & 0.4 \\
\hline
\end{tabular}

The probability of $\mathrm{HO}$ in traditional and composed technique is presented with respect to HT and Vsu, Vsu and Psu. Eventually the comparative analysis is shown for each parameter and it is observed that the proposed system is capable of reducing the HO probability in terms of velocity, power and channel hold time. The average probability is reduced by 0.1 by and the overall performance in $\mathrm{CRN}$ is enhanced with the reduction in unnecessary handover occurrence.

\section{CONCLUSION}

In CRN the ping pong effect is overcome by novel strategy, the technique implemented is Fuzzy Inference System and Neural networks are amalgamated to utilize their advantages.

The proposed model resulted into two ANFIS controllers which are used to compute the network parameters. The comparative analysis of conventional fuzzy logic controller and proposed ANFIS controller ensured the efficacy of the proposed work and the handover probability experienced overall reduction of 0.1 in the total average probability. Reduced probability indicated the effectiveness of the proposed ANFIS in terms of better handoff and low ping pong effect.

Though the proposed approach is proved to be better in reducing the HO probability but still there is a scope of enhancing this mechanism. Following aspects can be taken into consideration to improve the performance and efficiency of ANFIS model, the complexity of using two ANFIS model can be reduced and reliance on parameters can be increased by introducing the concept of mobility.

\section{REFERENCES}

[1] Sarah Javed and Bushra Naeem, "Reduction of Ping-Pong Effect in Cognitive Radio Spectrum Handoffs using Fuzzy Logic Based Inference", Published in 2018 UKSimAMSS 20th International Conference on Modelling \& Simulation, IEEE, Pg. No: 19-22, June 2019. 
Implementation of ANFIS Algorithm for Efficient Spectrum Hands-offs in Cognitive Radio

Networks

[2] Giupponi, Lorenza, and Ana I Pérez-Neira, "Fuzzy-based spectrum handoff in cognitive radio networks", Published in 3rd International Conference on Cognitive Radio Oriented Wireless Networks and Communications (Crown Com 2018) IEEE, Pg. No: 26-31, 2018.

[3] Ahmed, Ejaz, et al, "Channel assignment algorithms in cognitive radio networks Taxonomy, open issues, and challenges", Published in IEEE Communications Surveys \& Tutorials 18.1, Pg. No: 795-823, 2014

[4] Shimaa Ali and Mona Shokair. "Backup Channel Selection Approach for spectrum hands off in cognitive radio networks", published in IEEE $13^{\text {th }}$ international conference on computer engineering \& systems, 14 Feb 2019.

[5] Moniul Hossain and Jiang Xie, "Covert Spectrum Handoff attack in spectrum Hands-off processed in the cognitive Radio networks", Published in IEEE Global commination's conference, 21 Feb 201.

[6] Manav Aggarwal and T. Velmurugan, "Probability based centralized device for spectrum hands off in cognitive radio networks", published in IEEE on AI and cognitive computing for commination and network,20 Feb 2019.

[7] Prince Semba Yawada \& Mai Trung Dong, "Intelligent process of spectrum handoff/mobility in Cognitive radio Networks", Published in Journal of electrical and computer science, 13March 2019.

[8] Ozger, Mustafa, and Ozgur B. Akan, "On the utilization of spectrum opportunity in cognitive radio networks", Published in IEEE Communications Letters 20.1, Pg. No:157160, 2015.

[9] Haykin, Simon, "Cognitive radio: brain-empowered wireless communications", Published in IEEE journal on selected areas in communications 23.2, Pg. No: 201-220, 2018.

[10] Aguilar-Gonzalez, Rafael, et al. "Reducing spectrum handoffs and energy switching consumption of madm-based decisions in cognitive radio networks", Published in IEEE journal on Mobile Information Systems, 2016.

[11] A. Singh, M. Bhatnagar, and R. Malik, "Performance of an improved energy detector in multi-hop cognitive radio networks", IEEE Trans. VehTechnol., Feb-2015

[12] Prince Semba Yawada and Mai Trung Dong, "Intelligent Process of Spectrum Handoff/Mobility in Cognitive Radio Networks", Published in Journal of Electrical and Computer Engineering, Vol.4 12 pages, 2019

[13] S. Zahed, I. Awan, and A. Cullen, "Analytical modeling for spectrum handoff decision in cognitive radio networks", Simulation Modelling Practice, vol. 38, pp. 98-114, 2013.

[14] V. R. G. Vinay S. Prabhavalkar, "A Novel Approach to Reduce the Spectral Ping-Pong Effect for the Mobility Management Framework in a Cognitive Radio Cellular Network", ICGST-ACSE, vol. 15, 2015. 